\title{
The Effects of Exogenous Pyridoxal-5-phosphate on Seedling Growth and Development of Wheat under Salt Stress
}

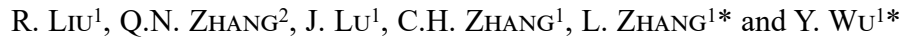 \\ ${ }^{1}$ Chengdu Institute of Biology, Chinese Academy of Sciences, Chengdu, 610041, China \\ ${ }^{2}$ Renqiu Affiliated School of Beijing Normal University, Renqiu, 062550, China
}

(Received 13 December 2018; Accepted 16 April 2019;

Communicated by A. Goyal)

\begin{abstract}
Salt stress is one of the major abiotic stress which severely limits plant growth and reduces crop productivity across the world. In the present study, the effects of exogenous pyridoxal-5-phosphate (vitamin $\mathrm{B}_{6}, \mathrm{VB}_{6}$ ) on seedling growth and development of wheat under salt stress were investigated. The results showed that exogenous application of pyridoxal-5-phosphate $\left(\mathrm{VB}_{6}\right)$ significantly increased the RWC, biomass, the concentration of photosynthetic pigments, proline, the activities of superoxide dismutase (SOD), catalase (CAT), peroxidase (POD), together with decreasing the content of Malondiadehyde (MDA) and hydrogen peroxide $\left(\mathrm{H}_{2} \mathrm{O}_{2}\right)$ in wheat leaves under salt stress. Meanwhile, the transcript level of P5CR, P5CS, SOD, TaSOS1 and TaSOS4 were also up-regulated after treatment with pyridoxal-5-phosphate. $\mathrm{VB}_{6}$ acts as a signal in regulating the activities of plant antioxidant enzymes and SOS pathway to improve resistance to salt stress. The current study results may give an insight into the regulatory roles of $\mathrm{VB}_{6}$ in improving salt stress and $\mathrm{VB}_{6}$ could be an easily and effective method to improve salt-stress tolerance to wheat in the field condition. It is urgency to understand the molecular mechanism of $\mathrm{VB}_{6}$ to enhance the salt tolerance of wheat in the next work.
\end{abstract}

Keywords: pyridoxol-5-phosphate, vitamin $\mathrm{B}_{6}$, salt stress, wheat, Triticum aestivum

\section{Introduction}

Salinity soil is a worldwide problem threatening crop production and leads to reduction in the economic yield of a wide variety of crops in mostly sea costal area across the world. (Askari et al. 2006; Munns and Gilliham 2015). In previous study, it was estimated that 150 million ha cultivated land out of 0.77 billion ha (about $5 \%$ ) was affected by soil salinity (Askari et al. 2006). On the other hand, about 27 million ha of salted land in China, out of these 0.06 billion ha is cultivated land (accounting for $8.5 \%$ ) affecting by salt stress (Li et al. 2005; Wang et al. 2011).

Plant growth and development can be affected by salinity stress at any time during the crop life cycle (Arzani 2008). Salt soil will disrupt homeostasis, physiological and biochemical processes in cells (Gururani et al. 2013). It also impacts cell membrane stability, photosynthesis, the concentration of chlorophyll, protein content and relative water con-

*Corresponding authors; E-mails: 13555809468@163.com; wuyugood@126.com 
tent (RWC) in plants (Talaat and Shawky 2014). Excessive amounts of sodium $\left(\mathrm{Na}^{+}\right)$and $\mathrm{Cl}^{-}$in soil can adversely affected on metabolism of plant cells (Shalata and Neumann 2001). Salt-stress conditions also lead to accumulation of reactive oxygen species (ROS) and lipid peroxidation products in plant roots, stems and leaves (Shalata and Neumann 2001). ROS like superoxide radical $\left(\mathrm{O}^{2-}\right)$, hydrogen peroxide $\left(\mathrm{H}_{2} \mathrm{O}_{2}\right)$ and hydroxyl radicals $\left(\mathrm{OH}^{-}\right)$cause lipid peroxidation of plant cell membranes (Gururani et al. 2013). To mitigate its (ROS) damage to cells, plants activate a range of enzymatic and non-enzymatic defense systems to alleviate cellular damage under salinity stress conditions (Hasanuzzaman et al. 2011).

Many studies have shown that applying suitable concentration of endogenous substances (plant growth regulator) can improve salt tolerance in plants. Such as jasmonic acid (JA) (Qiu et al. 2014), Salicylic Acid (SA ) (Arfan et al. 2007), ascorbic acid (AsA) (Shalata and Neumann 2001), mannitol (Seckin et al. 2009), and 24-epibrassinolide (Dong et al. 2017) can inhibit accumulation of active oxygen species (ROS) and alleviate $\mathrm{NaCl}$ damages to plants. Therefore, exogenous application of plant growth regulator gives an effective way to enhance plant resistance in abiotic stress, but the regulating mechanism of such endogenous substances in plant growth remains unclear.

Vitamin $\mathrm{B}_{6}$ is one of the essential cofactors for numerous metabolic enzymatic reactions and is considered as a potent antioxidant in plant organisms (Tambasco-Studart et al. 2005; Titiz et al. 2006). VB $_{6}$ include pyridoxine-5-phosphate (PLP) which constituting the active form of $\mathrm{VB}_{6}$ (Herrero and Daub 2007). As the active intracellular form, PLP has multiple roles of a versatile cofactor that almost exclusively functions in the metabolism of amino compounds. Wang et al. (2004) reported that pyridoxal kinase is a salt tolerance determination important for the regulation of $\mathrm{Na}^{+}$and $\mathrm{K}^{+}$homeostasis in plants. The new feature of $\mathrm{VB}_{6}$ as a ROS scavenger, and its potential ability to increase resistance to both biotic and abiotic stresses, has opened up new directions of plant $\mathrm{VB}_{6}$ research in photosynthesis and pathogen-response (Mooney and Hellmann 2010). Wheat (Triticum aestivum L.) is the second major crop planted in the world (Bhardwaj 2010). Saline soil limits wheat production by reducing plants biomass, RWC, photosynthetic pigments, proline, the activities of peroxidase in wheat leaves as other crops (Egamberdieva 2009; Qiu et al. 2014). Therefore, the objective of this study was to investigate the regulatory functions and mechanism of exogenous $\mathrm{VB}_{6}$ in wheat seedlings under salt stress.

\section{Materials and Methods}

The wheat seeds (Triticum aestivum L.) used in this study is Chuanyu23 (Fig. S4*) from Chengdu Institute of Biology, Chinese Academy of Sciences, which is one of the varieties certificated by Sichuan province in 2003. Wheat seeds were surface sterilized with $10 \%$ (v/v) sodium hypochlorite solution for $10 \mathrm{mins}$, then vigorously rinsed with distilled water $(>200 \mathrm{ml} /$ per time $)$ for 5 times. Sterilized seeds were sown in plug tray $(10 \mathrm{~cm} \times 12 \mathrm{~cm}$ pot) and arranged in illumination incubator with $25^{\circ} \mathrm{C} 16 \mathrm{~h}$ light $\left(600 \mu \mathrm{mol} \mathrm{m} \mathrm{m}^{-2} \mathrm{~s}^{-1}\right)$,

*Further details about the Electronic Supplementary Material (ESM) can be found at the end of the article. 
$18{ }^{\circ} \mathrm{C} 8 \mathrm{~h}$ darkness, $65 \%$ relative humidity (Dong et al. 2017). Seven days after sowing, plants at the same growth stage were selected and transplanted to containers filled with Hoagland solution. Plants were added with water or with pyridoxine-5-phosphate $\left(\mathrm{VB}_{6}\right)$. The treatments is given as follows: (1) $0 \mathrm{mM} \mathrm{NaCl}+0 \mathrm{mM} \mathrm{VB}_{6}$ (CK); (2) $200 \mathrm{mM}$ $\mathrm{NaCl}+0 \mathrm{mM} \mathrm{VB}_{6}(\mathrm{NaCl})$; (3) $0 \mathrm{mM} \mathrm{NaCl}+100 \mathrm{mM} \mathrm{VB}_{6}$; (4) $200 \mathrm{mM} \mathrm{NaCl}+100 \mathrm{mM}$ $\mathrm{VB}_{6}(\mathrm{NaCl})$. The nutrient solution was adjusted to $\mathrm{pH}$ 6.5-6.8. The treatment solution was changed daily to maintain constant $\mathrm{NaCl}$ concentration. The plants were sampled at $7 \mathrm{ds}$ after treatment.

\section{Measurement of biomass and physiological index in wheat seedlings}

The current study measured height of seedlings, root length, fresh and dry weight of Chuanyu 23 seedlings after $14 \mathrm{~d}$ of germination in Figure S1. The seedlings were dried at $80{ }^{\circ} \mathrm{C}$ for 2 days for dry weight measurement, and their final dry weights were measured in Figure S2. For relative water content was calculated (Fig. S3) as follow: $\mathrm{RWC}(\%)=(\mathrm{FW}-\mathrm{DW}) / \mathrm{FW} \times 100$ (Dong et al. 2017). The chlorophyll content (Song et al. 2016) was determined (Fig. S3). Lipid peroxidation was evaluated by measuring malondialdehyde content (MDA). According to the method (Rao and Sresty 2000), MDA content was determined (slightly modified). $\mathrm{H}_{2} \mathrm{O}_{2}$ content was determined according to Shi et al. (2005). Proline concentration (Bates et al. 1973) was determined. Superoxide dismutase (SOD) activity (Giannopolitis and Ries 1977) was measuring at $560 \mathrm{~nm}$. Peroxidase (POD) activity (Kochba et al. 1977) was measured by the increase in absorbance at $470 \mathrm{~nm}$. Catalase (CAT) activity (Cakmak and Horst 1991) was measured as the decline in absorbance at $240 \mathrm{~nm}$. RNA extraction use TransZol ${ }^{\mathrm{TM}}$ Up Plus RNA kit (from TransGen Biotech). The cDNA was synthesized from total RNA using a first strand cDNA synthesis kit (from TransGen Biotech). Primers used for the relative quantification of biosynthetic gene transcripts in Table S1.

\section{Statistical analysis}

SPSS 20.0 statistical analysis software, Graphpad software 5.0 and Microsoft Excel 2013 software were used to analyze all the experimental data in this study. One-way analysis of variance (ANOVA) was conducted to evaluate the variance and significance between groups. Differences between treatments were separated by the least significant difference (LSD) test at 0.05 level.

\section{Result}

\section{The biomass index of wheat seedling}

The current study resulted that salt stress suppressed the growth of the wheat seedlings. The biomass of wheat roots and shoots were reduced (Fig. 1, Figs S1, S2) under salt stress. However, adding $200 \mathrm{mM} \mathrm{VB}_{6}$ were mitigate wheat plant growth inhibition by salt 

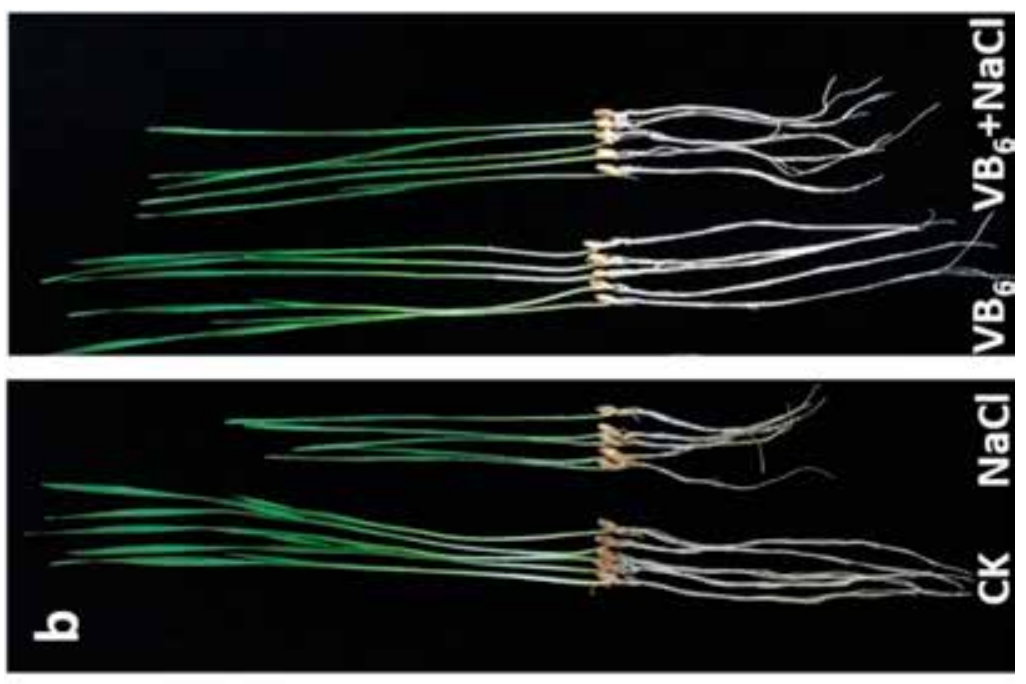

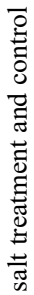
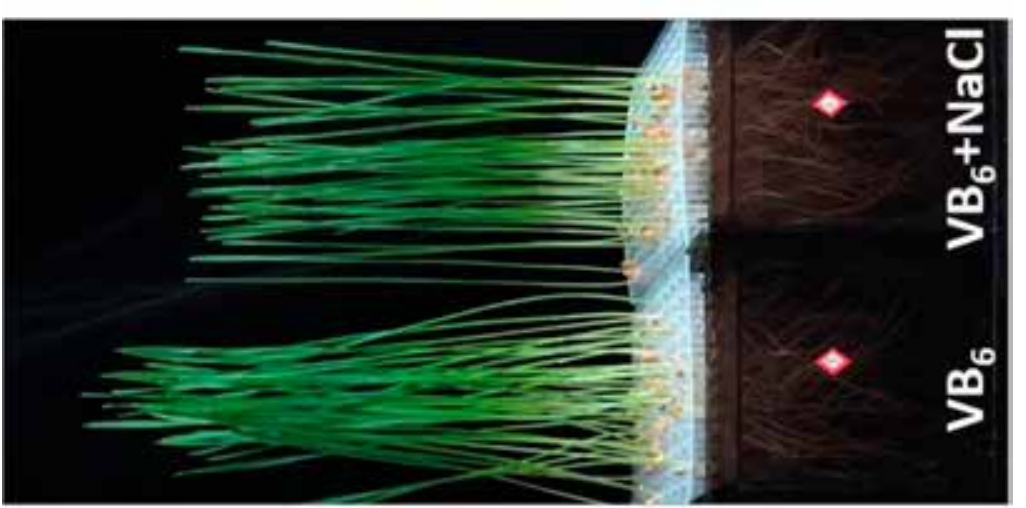

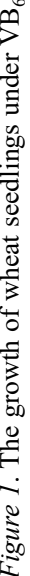

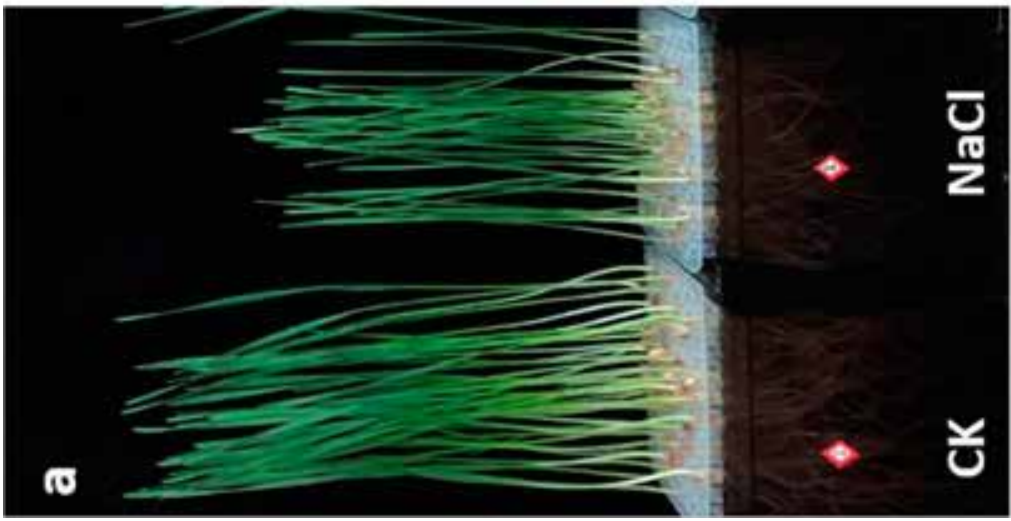

Cereal Research Communications 47, 2019 

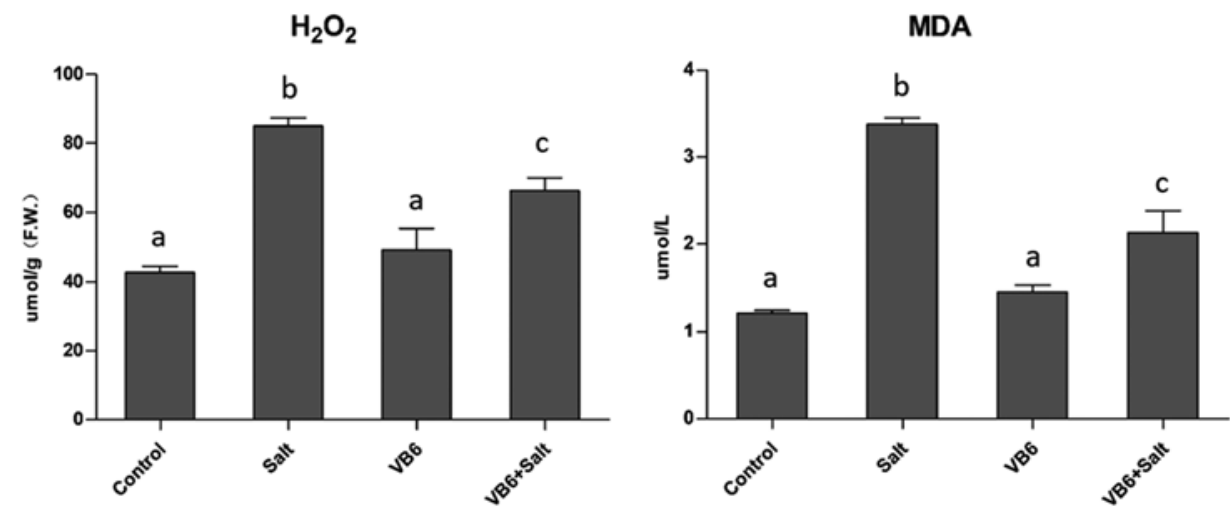

Figure 2. The contents of $\mathrm{H}_{2} \mathrm{O}_{2}$ and MDA in wheat leafs, bars with different letters are significantly different at $5 \%$ level $(\mathrm{p}<0.05)$

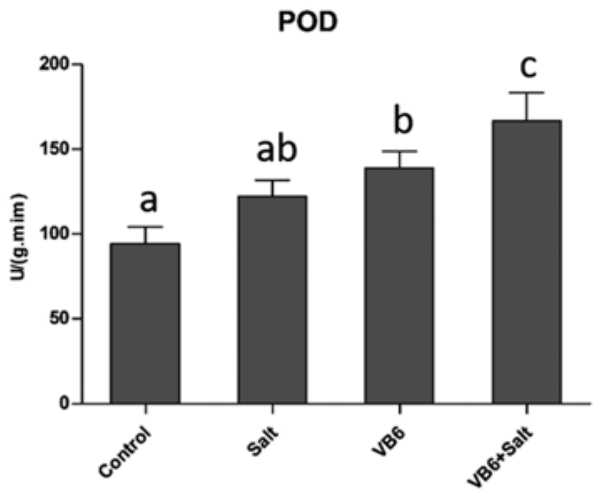

SOD

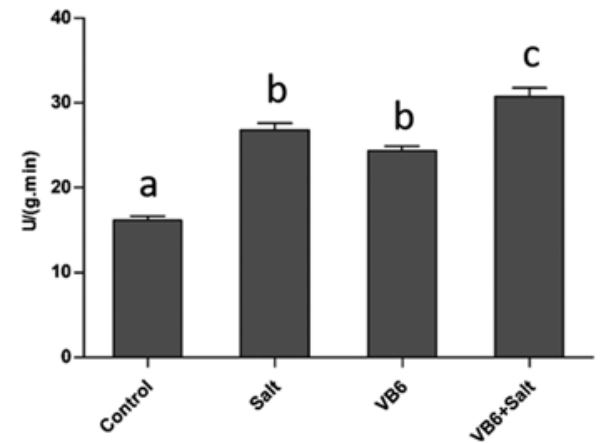

CAT

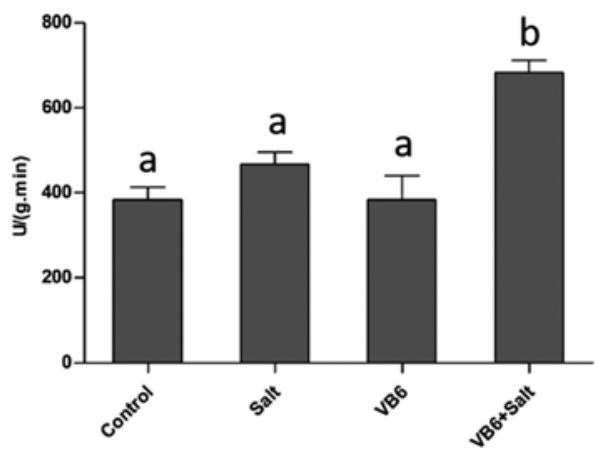

$S O D$

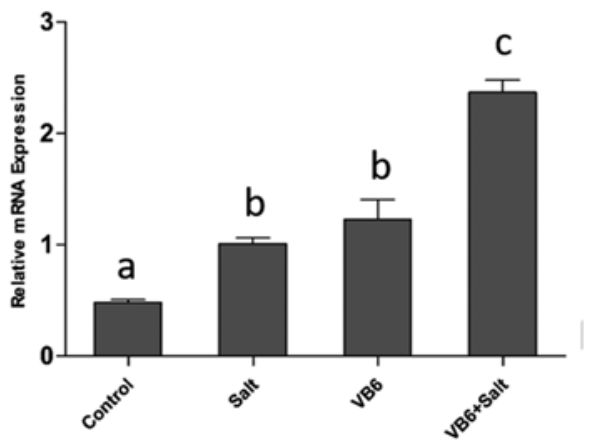

Figure 3. The enzyme activity of POD, CAT and SOD in the wheat seedlings, and the SOD expression patterns, bars with different letters are significantly different at $5 \%$ level $(\mathrm{p}<0.05)$ 
stress (Figs S1, S2). This results showed that exogenous application of $\mathrm{VB}_{6}$ effectively alleviated growth suppression in the wheat seedlings under salt stress.

\section{Photosynthetic pigments and relative water content of wheat seedling}

With salt treatment the content of chlorophyll a, chlorophyll b, carotenoid and RWC significantly decreased in wheat seedlings (Fig. S3). The $\mathrm{VB}_{6}+$ Salt treatment increased photosynthetic pigments and RWC content. These results suggest that exogenous $\mathrm{VB}_{6}$ can alleviate the degradation of chlorophyll and the loss of cell water in wheat under salt stress.

\section{Wheat seedling lipid peroxide}

The content of $\mathrm{H}_{2} \mathrm{O}_{2}$ and MDA in plant tissues is an important indicator to measure the reactive oxygen species and damage of plant cells under salt stress condition. The contents of $\mathrm{H}_{2} \mathrm{O}_{2}$ and MDA increased significantly in Chuanyu23 seedlings with salt or salt $+\mathrm{VB}_{6}$ treatment (Fig. 2). For the salt treatment, the $\mathrm{H}_{2} \mathrm{O}_{2}$ and MDA contents were about 2 and 2.8-fold higher compare with control. But significantly decreased with the application of $\mathrm{VB}_{6}$ (Fig. 2). The result demonstrates that application $\mathrm{VB}_{6}$ under salt stress is beneficial to reduce the reactive oxygen species in plant cell and diminish the damage of plant cell membrane.

\section{Activity of peroxidase in wheat seedling}

After application of $\mathrm{VB}_{6}$, the enzyme activity of POD, CAT and SOD in the wheat seedlings was higher than that in the salt treatment alone (Fig. 3). When the wheat seedlings were treated with the $200 \mathrm{mM} \mathrm{NaCl}$, the enzyme activity of POD, CAT and SOD increased. But the increase was even more significant after application of $\mathrm{VB}_{6}$. The results indicated that application $\mathrm{VB}_{6}$ under salt stress can effectively increase the activity of POD, CAT and SOD enzymes, and remove more free radicals under salt stress, then reduce cell membrane damage, and improve the plant resistance to salt stress.

To further explore the mechanism of $\mathrm{VB}_{6}$ in improving plant resistance to salt stress, RT-qPCR was conducted to analyze the expression patterns of the antioxidant gene $S O D$. Interestingly, the transcript level of SOD increased significantly by 2.4 -fold in the salt $+\mathrm{VB}_{6}$ treatment compared to the salt treatment (Fig. 3), which is consistent with the result of SOD enzyme activity. And application of $\mathrm{VB}_{6}$ significantly up-regulated the SOD gene expression, when without salt stress conditions in wheat plants. So we infer that $\mathrm{VB}_{6}$ might act as a signal in regulating the expressions of some peroxidase genes, such as SOD under salt stress. 


\section{Osmotic regulator of wheat seedling}

Wheat seedling growth in salt stress has higher proline content (about 2.8 times) than control (Fig. 4). Similarly, after application of $\mathrm{VB}_{6}$ the content of proline in salt-stress also increased by 2.5 times compared with non-salt group. $\mathrm{VB}_{6}$-treated seedling accumulated more proline than salt stressed alone, however, it did not increase significantly. High content of proline can protect wheat seedlings and alleviated the growth inhibition caused by salt stress.

The expression of proline biosynthesis genes (P5CR and P5CS) were also investigated by quantitative real-time PCR (RT-qPCR). The P5CR and P5CS are two of key enzymes in the proline synthesis, which can reflect the changes of proline content in wheat plants. The expressions of P5CR and P5CS in the wheat seedlings with $\mathrm{VB}_{6}$ treatment were significantly up-regulated under salt stress (Fig. 4), which is consistent with the above result that application of VB6 can promote the proline synthesis by up-regulating the expression of P5CR and P5CS. For same genes, the expression level of $\mathrm{VB}_{6}$ treated seedlings has

pro.

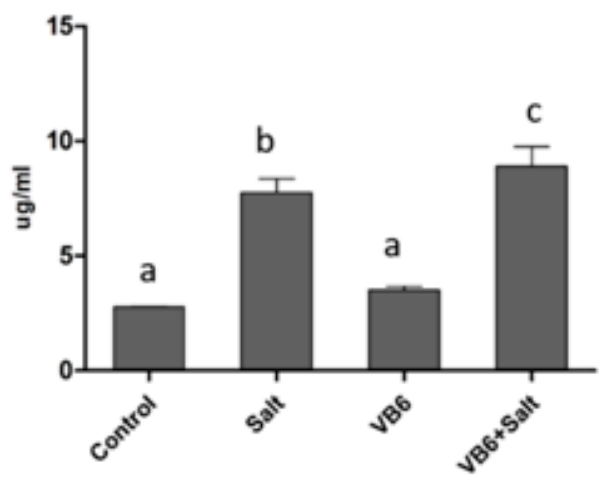

P5CR

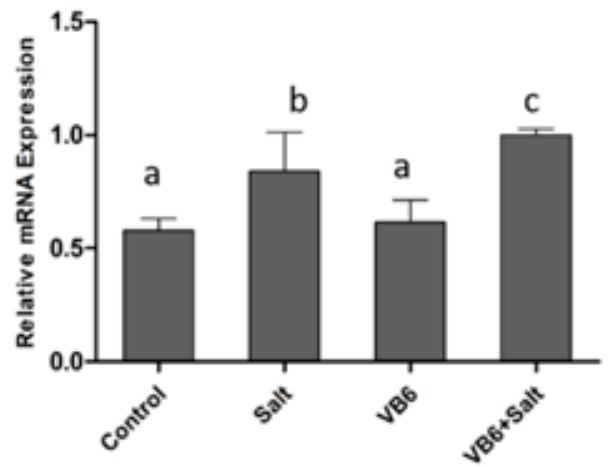

P5CS

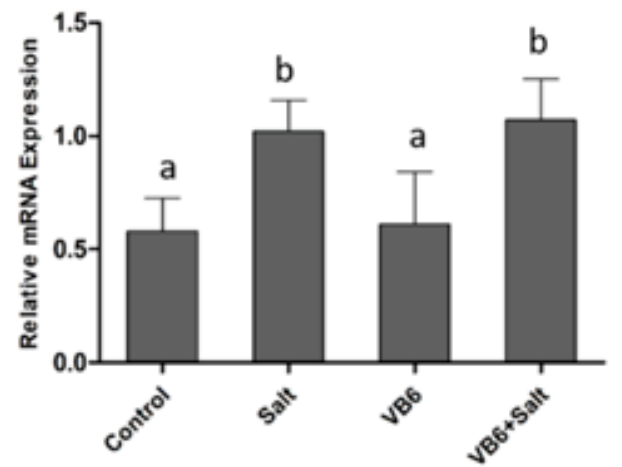

Figure 4. The content of proline in wheat seedlings and the expression of P5CR and P5CS, bars with different letters are significantly different at $5 \%$ level $(\mathrm{p}<0.05)$ 
changed not significantly compared to the control plants (Fig. 4). Therefore, we suggest that $\mathrm{VB}_{6}$ pretreatment will up-regulate the expression of P5CS and P5CR in wheat seedlings exposed to $200 \mathrm{Mm} \mathrm{NaCl}$ treatments.

\section{Expression of TaSOS1 and TaSOS4 in wheat}

SOS1 and SOS4 are two genes related to Salt Overly Sensitive (SOS) pathway, which plays a vital role in exclusion $\mathrm{Na}^{+}$at cellular level, and mitigated osmotic stress caused by high salt condition. SOS1 is a $\mathrm{Na}^{+} / \mathrm{H}^{+}$antiporter that regulate the $\mathrm{Na}^{+}$transport in salt stress (Xu et al. 2008). SOS4 is a vital cofactor for enzymes in the cell, and pyridoxal kinase encodes by SOS4 to participate in pyridoxal-5-phosphate biosynthesis in the cell. It also involved in regulation of ion transport of cells in salt stress (Mahajan et al. 2008). The quantitative expressions of TaSOS1 and TaSOS4 genes in wheat leaves under salinity stress were investigated in current study (Fig. 5). $\mathrm{VB}_{6}$ treated seedlings has up-regulate in TaSOS1 and TaSOS4 expression (Fig. 5), but not significant. Applied $\mathrm{VB}_{6}$ in salt-stressed nutrient solution, seedlings recorded about 2.1-fold higher TaSOS1 expression compared to the salt-stressed plants. Similar to the TaSOS1 gene response, wheat plants increased significant (about twice) in the expression levels of TaSOS4 in salt stress. $\mathrm{VB}_{6}$ enhanced the expression of TaSOS1 and TaSOS4 in wheat leafs under salt stress.

\section{TaSOS1}

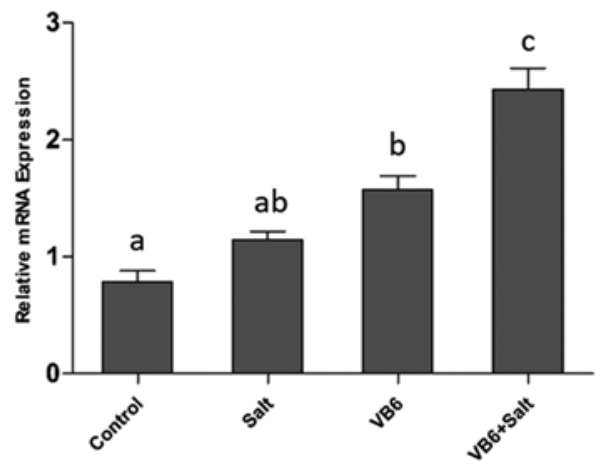

TaSOS4

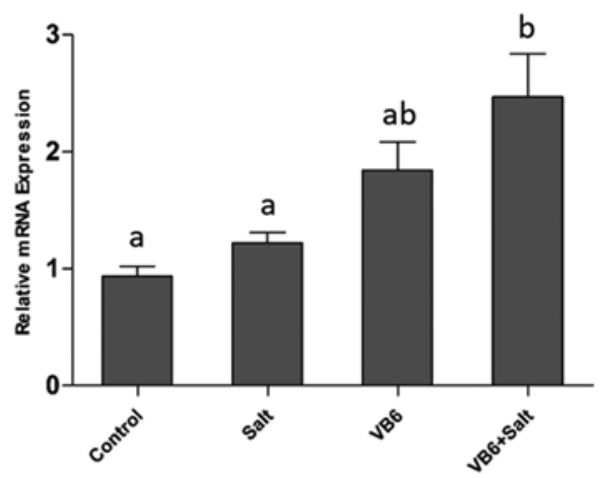

Figure 5. The expression of TaSOS1 and TaSOS4, bars with different letters are significantly different at 5\% level $(\mathrm{p}<0.05)$

\section{Discussion}

The biomass and growth development in wheat

The responses of cultivated crop species to salinity in terms of growth and yield are the ultimate expression of several interacting physiological and biochemical parameters (Almansouri et al. 2001). High concentrations of salinity in the soil severely restrict plant growth and development (Ashraf and Foolad 2013). The current study found similar 
results that the length of wheat roots and seedlings were significantly decreased at 200 $\mathrm{mM} \mathrm{NaCl}$-stress. However, application of $\mathrm{VB}_{6}$ were mitigate growth inhibition under salt stress (Figs S1, S2).

Photosynthesis is the most important process that directly or indirectly influencing the growth and survival of every organism (flora and fauna) (Gururani et al. 2013). Therefore, it cannot be examined simply as an isolated phenomenon but must be studied within the context of whole-plant regulation. Salinity-stress effects on crop growth are manifested by impairment of photosynthetic capacity (Brini et al. 2007). Salinity also reduced wheat relative growth rate, photosynthetic rate in stems and leaves during whole crop cycle from seed germination to crop maturity (El-Hendawy et al. 2005). An interesting aspect of PLP-dependent enzymes is also impact on the biosynthesis of phytohormones which are key regulators in plant development (Mooney and Hellmann 2010). The current study found that the content of chlorophyll and carotenoid were significantly reduced in wheat seedlings (Fig. S3). Photosynthetic pigments degradation was relieved by $\mathrm{VB}_{6}$ treatment.

\section{Regulate osmotic system in wheat}

Osmotic adjustment can alters the relationship between cell protoplast volumes (approximated by RWC) (Brini et al. 2007). The $\mathrm{VB}_{6}+$ salt treatment showed increased RWC content compared with CK (Fig. S3). Proline has been shown to accumulate in plant tissues under various stress conditions (Gururani et al. 2013). The proposed function of the accumulated proline in osmosis regulation has an adaptive mechanism to environmental stress and salinity (El-Sayed et al. 2014). Previous research reported that the proline concentration significantly increased in the leaf of all cultivars under increasing salt tolerance (Moradi and Ismail 2007). The current results show that wheat seedling growth in salt stressed condition have higher proline content (Fig. 4). $\mathrm{VB}_{6}$ treated seedling accumulation more proline. Two key enzymes in proline biosynthesis $\Delta^{1}$-pyrropline-5-carboxylate syntheses (P5CS) and $\Delta^{1}$-pyrropline-5-carboxylate reductase (P5CR) (Porcel et al. 2004; Zhang et al. 2014) were estimated in the current study. And the result suggested that $\mathrm{VB}_{6}$ enhances the tolerance of wheat seedlings to salt stress, synthesizes more proline by increasing the expression of proline synthesis gene (P5CS and P5CR), and enhances the osmotic regulation ability of plants to protect them from damage.

\section{Activate peroxidase system in wheat}

Cellular redox state is an important factor which regulates the key process in growth and development as well as stress tolerance (Jisha et al. 2013). In plant, the salt effect on the enzymes might be involved in the dark reactions of photosynthesis and could result in the generation of excess ROS with subsequent damage to lipid membranes, as demonstrated by the high levels of MDA and severe leaf damage in this cultivar (Moradi and Ismail 2007). The current study suggests that salinity-induced oxidative damage in wheat seedlings, as indicated by the higher levels of $\mathrm{H}_{2} \mathrm{O}_{2}$ and MDA, is probably due to the inhibi- 
tion or insufficient induction of the antioxidant defense (Hasanuzzaman et al. 2011). The current study showed that $200 \mathrm{mM} \mathrm{NaCl}$ stress increased the content of $\mathrm{H}_{2} \mathrm{O}_{2}$ and MDA in seedlings. But significantly reduced after the application of $\mathrm{VB}_{6}$ (Fig. 2). Hydrogen peroxide levels in wheat were significantly higher in control group under all the applied salt-stress treatments than in the $\mathrm{VB}_{6}$ treated seedlings. $\mathrm{VB}_{6}$ enabled wheat seedlings to maintain a higher proline content and lower $\mathrm{MDA}, \mathrm{H}_{2} \mathrm{O}_{2}$ accumulation in wheat. The present study results indicated that use $\mathrm{VB}_{6}$ can effectively reduce the damage of $\mathrm{NaCl}$ stress to wheat seedlings by reducing $\mathrm{H}_{2} \mathrm{O}_{2}$ and MDA content.

The antioxidant enzymes of the cell have the ability to remove the free radicals produced during abiotic stress conditions. These enzymes also protect the membranes and DNA from damage (Gururani et al. 2013). The specific activity of SOD significantly increased in the $\mathrm{VB}_{6}$ treated seedlings growing under $\mathrm{NaCl}$ stresses. Plant tolerance to these stresses correlated with the increased expression levels of ROS-scavenging enzymes $\mathrm{SOD}, \mathrm{CAT}$ and POD, suggesting that $\mathrm{VB}_{6}$ treated wheat seedling triggered salt stress related defense pathways under high salinity conditions. The mRNA expression of SOD in wheat plants growing under salt-stress with $\mathrm{VB}_{6}$ conditions increased. The present experimental result was consistent with the RT-qPCR results where the relative mRNA expression levels of the anti-oxidative pathway genes were more pronounced in the $\mathrm{VB}_{6}$ applied wheat than in the non- $\mathrm{VB}_{6}$. Based on the finding, it was suggested that $\mathrm{VB}_{6}$ could play an important role in oxidative stress injury of wheat leaves grown in stressed condition. Possibly, the protective effect of $\mathrm{VB}_{6}$ is more related to reduced active oxygen species (ROS) damage to essential proteins and/or nucleic acids. The current study suggest that exogenous application of $200 \mathrm{mM} \mathrm{VB}_{6}$ could alleviate salt-induced oxidative damage by enhancing antioxidant enzyme activities in the seedling.

\section{Activate SOS pathway in wheat}

High $\mathrm{Na}^{+}$concentration in the cytosol is detrimental to plant growth and leaves are usually more susceptible to $\mathrm{Na}^{+}$toxicity. Further to control the transport of $\mathrm{Na}^{+}$and $\mathrm{Cl}^{-}$is very critical for salinity tolerance in plants. On the other hand, the excess ions will enter in cell and accumulate to toxic levels in the older transpiring plant leaves (Munns et al. 2010). The Current research had reported that the $\mathrm{Na}^{+} / \mathrm{H}^{+}$antiporters in cytoplasmic membrane plays an important role in plant resistance to salt stress (Chen et al. 2008). Salt Overly Sensitive (SOS) pathway plays a vital role in exclusion $\mathrm{Na}^{+}$at cellular level by regulate $\mathrm{Na}^{+} / \mathrm{H}^{+}$antiporters, and mitigated osmotic stress caused by high salt condition (Shi et al. 2003; Xu et al. 2008). Previous study found that over expression of SOS1 can reduce the accumulation of sodium ions and enhance the salt tolerance in transgenic Arabidopsis (Shi et al. 2003). The TaSOS1 protein is induced by salt treatment and contributes to plasma membrane $\mathrm{Na}^{+} / \mathrm{H}^{+}$exchange (Xu et al. 2008). Whereas the present study measured the expression of TaSOS1 and TaSOS4 in wheat that are related to SOS pathway. The current study result shown that application of salinity stress lowered TaSOS1 and TaSOS4 genes expression compared with $\mathrm{VB}_{6}$ treated in wheat plants (Fig. 5). With the salt stress, application of $\mathrm{VB}_{6}$ markedly enhanced the expression of 
TaSOS1 and TaSOS4 in wheat leaves. It means $\mathrm{VB}_{6}$ can improve the salt tolerance of wheat plants by active SOS pathway in plant and regulate the ion transporters to exclude the excessive ion to protect plant in salt stressed conditions. SOS4 have also been characterized and reported that SOS4 may be involved the pyridoxal-5-phosphate biosynthesis which encodes a pyridoxal kinase (Ramezani et al. 2013). It is an active form of vitamin $\mathrm{B}_{6}$ (Mahajan et al. 2008). Like previous reports, the application of $\mathrm{VB}_{6}$ markedly enhanced the expression of TaSOS4 in wheat leaves in the present study. Further adding the $\mathrm{VB}_{6}$ to up-regulate the expression of SOS4 in plant cells suggested that treatment with $\mathrm{VB}_{6}$ could be an easily and effective method to improve salt-stress tolerance to wheat.

In conclusion, the current result found that exogenous $\mathrm{VB}_{6}$ effectively alleviated plant growth inhibition. Application of $\mathrm{VB}_{6}$ is beneficial to reduce the reactive oxygen species in plant cell and diminish the damage of plant cell membrane under salt stress by increasing the activity of plant antioxidant enzymes (POD, CAT and SOD) to remove more free radicals caused by salt stress. Also, $\mathrm{VB}_{6}$ might act as a signal in regulating the expressions of some peroxidase and osmotic regulator genes such as SOD, P5CS, P5CR, TaSOS1 and TaSOS4 under salt stress. The current study results give an insight into the regulatory roles of $\mathrm{VB}_{6}$ in improving salt stress by cross talking with peroxidase system, proline synthesis and SOS pathway.

\section{Acknowledgement}

This work was supported by the "13th Five-year Plan" for National Key Research and Development (2017YFD0100902). The grant number of the funding support is 2016YFD0102000.

\section{References}

Almansouri, M., Kinet, J.M., Lutts, S. 2001. Effect of salt and osmotic stresses on germination in durum wheat (Triticum durum Desf.). Plant Soil 231(2):243-254.

Arfan, M., Athar, H.R., Ashraf, M. 2007. Does exogenous application of salicylic acid through the rooting medium modulate growth and photosynthetic capacity in two differently adapted spring wheat cultivars under salt stress? J. Plant Physiol. 164(6):685-694.

Arzani, A. 2008. Improving salinity tolerance in crop plants: a biotechnological view. In Vitro Cell. Dev.-Pl. 44(5):373-383.

Ashraf, M., Foolad, M.R. 2013. Crop breeding for salt tolerance in the era of molecular markers and markerassisted selection. Plant Breeding 132(1):10-20.

Askari, H., Edqvist, J., Hajheidari, M., Kafi, M., Salekdeh, G.H. 2006. Effects of salinity levels on proteome of Suaeda aegyptiaca leaves. Proteomics 6(8):2542-2554.

Bates, L.S., Waldren, R.P., Teare, I.D. 1973. Rapid determination of free proline for water-stress studies. Plant Soil 39(1):205-207.

Bhardwaj, M., Maekawa, F., Niimura, Y., Macer, D.R.J. 2010. Ethics in food and agriculture: views from fao. Int. J. Food Sci. Tech. 38(5):565-577.

Brini, F., Hanin, M., Mezghani, I., Berkowitz, G.A., Masmoudi, K. 2007. Overexpression of wheat $\mathrm{Na}^{+} / \mathrm{H}^{+}$ antiporter TNHXI and $\mathrm{H}^{+}$-pyrophosphatase TVPl improve salt- and drought-stress tolerance in Arabidopsis thaliana plants. J. Exp. Bot. 58(2):301-308. 
Cakmak, I., Horst, W.J. 1991. Effect of aluminum on lipid-peroxidation, superoxide-dismutase, catalase, and peroxidase-activities in root-tips of soybean (glycine-max). Physiol. Plantarum 83(3):463-468.

Chen, L.H., Zhang, B., Xu, Z.Q. 2008. Salt tolerance conferred by overexpression of Arabidopsis vacuolar $\mathrm{Na}^{+} / \mathrm{H}^{+}$antiporter gene AtNHX1 in common buckwheat (Fagopyrum esculentum). Transgenic Res. 17(1):121-132.

Dong, Y.J., Wang, W.W., Hu, G.Q., Chen, W.F., Zhuge, Y., Wang Z.L., He, M.R. 2017. Role of exogenous 24-epibrassinolide in enhancing the salt tolerance of wheat seedlings. J. Soil Sci. Plant Nut. 17(3):554-569.

Egamberdieva, D. 2009. Alleviation of salt stress by plant growth regulators and IAA producing bacteria in wheat. Acta Physiol. Plant. 31(4):861-864.

El-Hendawy, S.E., Hu, Y.C., Schmidhalter, U. 2005. Growth, ion content, gas exchange, and water relations of wheat genotypes differing in salt tolerances. Aust. J. Agr. Res. 56(2):123-134.

El-Sayed, O.M., El-Gammal, O.H.M., Salama, A.S.M. 2014. Effect of ascorbic acid, proline and jasmonic acid foliar spraying on fruit set and yield of Manzanillo olive trees under salt stress. Sci. Hortic.-Amsterdam 176:32-37.

Giannopolitis, C.N., Ries, S.K. 1977. Superoxide dismutases 1. Occurrence in higher plants. Plant Physiol. 59(2):309-314.

Gururani, M.A., Upadhyaya, C.P., Baskar, V., Venkatesh, J., Nookaraju, A., Park, S.W. 2013. Plant growthpromoting rhizobacteria enhance abiotic stress tolerance in Solanum tuberosum through inducing changes in the expression of ROS-scavenging enzymes and improved photosynthetic performance. J. Plant Growth Regul. 32(2):245-258.

Hasanuzzaman, M., Hossain, M.A., Fujita, M. 2011. Nitric oxide modulates antioxidant defense and the methylglyoxal detoxification system and reduces salinity-induced damage of wheat seedlings. Plant Biotechnol. Rep. 5(4):353-365.

Herrero, S., Daub, M.E. 2007. Genetic manipulation of Vitamin B-6 biosynthesis in tobacco and fungi uncovers limitations to up-regulation of the pathway. Plant Sci. 172(3):609-620.

Jisha, K.C., Vijayakumari, K., Puthur, J.T. 2013. Seed priming for abiotic stress tolerance: an overview. Acta Physiol. Plant. 35(5):1381-1396.

Kochba, J., Lavee, S., Spiegelroy, P. 1977. Differences in peroxidase-activity and isoenzymes in embryogenic and non-embryogenic Shamouti orange ovular callus lines. Plant Cell Physiol. 18(2):463-467.

Li, B., Wang, Z.C., Sun, Z.G., Chen, Y., Yang, F. 2005. Resources and sustainable resource exploitation of salinized land in China. Agricultural Research in the Arid Areas 23(2):154-158.

Li, J., Sun, C.Y., Yu, N., Wang, C., Zhang, T.T., Bu, H.Y. 2016. Hexaconazole-Cu complex improves the salt tolerance of Triticum aestivum seedlings. Pestic. Biochem. Phys. 127:90-94.

Mahajan, S., Pandey, G.K., Tuteja, N. 2008. Calcium- and salt-stress signaling in plants: Shedding light on SOS pathway. Archives of Biochemistry and Biophysics 471(2):146-158.

Mooney, S., Hellmann, H. 2010. Vitamin $\mathrm{B}_{6}$ : Killing two birds with one stone? Phytochemistry 71(5-6): 495-501.

Moradi, F., Ismail, A.M. 2007. Responses of photosynthesis, chlorophyll fluorescence and ROS-scavenging systems to salt stress during seedling and reproductive stages in rice. Ann. Bot.-London 99(6):1161-1173.

Munns, R., Gilliham, M. 2015. Salinity tolerance of crops - what is the cost? New Phytol. 208(3):668-673.

Munns, R., Wallace, P.A., Teakle, N.L., Colmer, T.D. 2010. Measuring soluble ion concentrations $\left(\mathrm{Na}^{+}, \mathrm{K}^{+}, \mathrm{Cl}^{-}\right)$ in salt-treated plants. Methods in molecular biology (Clifton, N.J.) 639:371-382.

Porcel, R., Azcon, R., Ruiz-Lozano, J.M. 2004. Evaluation of the role of genes encoding for Delta(1)-pyrroline5-carboxylate synthetase (P5CS) during drought stress in arbuscular mycorrhizal glycine max and Lactuca sativa plants. Physiological and Molecular Plant Pathology 65(4):211-221.

Qiu, Z., Guo, J., Zhu, A., Zhang, L., Zhang, M. 2014. Exogenous jasmonic acid can enhance tolerance of wheat seedlings to salt stress. Ecotox. Environ. Safe. 104:202-208.

Ramezani, A., Niazi, A., Abolimoghadam, A.A., Babgohari, M.Z., Deihimi, T., Ebrahimi, M., Akhtardanesh, H., Ebrahimie, E. 2013. Quantitative expression analysis of TaSOS1 and TaSOS4 genes in cultivated and wild wheat plants under salt stress. Mol. Biotechnol. 53(2):189-197.

Rao, K.V.M., Sresty, T.V.S. 2000. Antioxidative parameters in the seedlings of pigeonpea (Cajanus cajan L. Millspaugh) in response to Zn and Ni stresses. Plant Sci. 157(1):113-128. 
Seckin, B., Sekmen, A.H., Turkan, I. 2009. An enhancing effect of exogenous mannitol on the antioxidant enzyme activities in roots of wheat under salt stress. J. Plant Growth Regul. 28(1):12-20.

Shalata, A., Neumann, P.M. 2001. Exogenous ascorbic acid (vitamin C) increases resistance to salt stress and reduces lipid peroxidation. J. Exp. Bot. 52(364):2207-2211.

Shi, H.Z., Lee, B.H., Wu, S.J., Zhu, J.K. 2003. Overexpression of a plasma membrane $\mathrm{Na}^{+} / \mathrm{H}^{+}$antiporter gene improves salt tolerance in Arabidopsis thaliana. Nat. Biotechnol. 21(1):81-85.

Shi, S.Y., Wang, G., Wang, Y.D., Zhang, L.G., Zhang, L.X. 2005. Protective effect of nitric oxide against oxidative stress under ultraviolet-B radiation. Nitric Oxide-Biol. Ch. 13(1):1-9.

Song, Y.L., Dong, Y.J., Tian, X.Y., Kong, J., Bai, X.Y., Xu, L.L., He, Z.L. 2016. Role of foliar application of 24-epibrassinolide in response of peanut seedlings to iron deficiency. Biol. Plantarum 60(2):329-342.

Talaat, N.B., Shawky, B.T. 2014. Protective effects of arbuscular mycorrhizal fungi on wheat (Triticum aestivum L.) plants exposed to salinity. Environ. Exp. Bot. 98:20-31.

Tambasco-Studart, M., Titiz, O., Raschle, T., Forster, G., Amrhein, N., Fitzpatrick, T.B. 2005. Vitamin B 6 biosynthesis in higher plants. P. Natl. Acad. Sci. USA 102(38):13687-13692.

Titiz, O., Tambasco-Studart, M., Warzych, E., Apel, K., Amrhein, N., Laloi, C., Fitzpatrick, T.B. 2006. PDX1 is essential for vitamin $\mathrm{B}_{6}$ biosynthesis, development and stress tolerance in Arabidopsis. Plant $\mathrm{J}$. 48(6):933-946.

Wang, H.B., Liu, D.C., Liu, C.G., Zhang, A.M. 2004. The pyridoxal kinase gene TaPdxK from wheat complements vitamin B-6 synthesis-defective Escherichia coli. J. Plant Physiol. 161(9):1053-1060.

Wang, J.L., Huang, X.J., Zhong, T.Y., Chen, Z.G. 2011. Review on sustainable utilization of salt-affected land. Acta Geographica Sinica 66 (5):673-684.

Xu, H.X., Jiang, X.Y., Zhan, K.H., Cheng, X.Y., Chen, X.J., Pardo, J.M., Cui, D.Q. 2008. Functional characterization of a wheat plasma membrane $\mathrm{Na}^{+} / \mathrm{H}^{+}$antiporter in yeast. Arch. Biochem. Biophys. 473(1):8-15.

Zhang, M., Huang, H., Dai, S.L. 2014. Isolation and expression analysis of proline metabolism-related genes in Chrysanthemum lavandulifolium. Gene 537(2):203-213.

Zhang, S.W., Gan, Y.T., Xu, B.L. 2016. Application of plant-growth-promoting fungi Trichoderma longibrachiatum T6 enhances tolerance of wheat to salt stress through improvement of antioxidative defense system and gene expression. Front. Plant. Sci. 7.

Zou, P., Li, K.C., Liu, S., Xing, R.G., Qin, Y.K., Yu, H.H., Zhou, M.M., Li, P.C. 2015. Effect of chitooligosaccharides with different degrees of acetylation on wheat seedlings under salt stress. Carbohyd. Polym. 126:62-69.

\section{Electronic Supplementary Material (ESM)}

Electronic Supplementary Material (ESM) associated with this article can be found at the website of CRC at https://akademiai.com/loi/0806

Electronic Supplementary Table S1. The primers for RT-qPCR

Electronic Supplementary Figure S1. Root length and shoot height of wheat seedlings in each treatment, bars with different letters are significantly different at $5 \%$ level $(\mathrm{p}<0.05)$

Electronic Supplementary Figure S2. Fresh/dry weight of wheat seedling roots and shoots, bars with different letters are significantly different at $5 \%$ level $(\mathrm{p}<0.05)$

Electronic Supplementary Figure S3. The content of chlorophyll a, chlorophyll b, carotenoid and relative water content in wheat seedlings, bars with different letters are significantly different at $5 \%$ level $(\mathrm{p}<0.05)$

Electronic Supplementary Figure S4. The pedigree of the Chuanyu23 